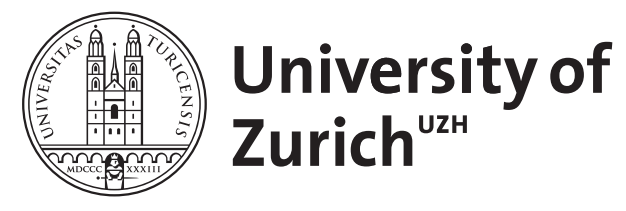

Zurich Open Repository and Archive

University of Zurich

University Library

Strickhofstrasse 39

CH-8057 Zurich

www.zora.uzh.ch

Year: 1992

Ein Programm-System zur Auswertung von Doppler-Ultraschall-Messungen

König, Volker ; Huch, Renate ; Huch, Albert

DOI: https://doi.org/10.1515/bmte.1992.37.s2.176

Posted at the Zurich Open Repository and Archive, University of Zurich

ZORA URL: https://doi.org/10.5167/uzh-154054

Journal Article

Published Version

Originally published at:

König, Volker; Huch, Renate; Huch, Albert (1992). Ein Programm-System zur Auswertung von DopplerUltraschall-Messungen. Biomedizinische Technik. Biomedical engineering, 37(s2):176-177.

DOI: https://doi.org/10.1515/bmte.1992.37.s2.176 


\title{
Ein Programm-System zur Auswertung von Doppler-Ultraschall-Messungen
}

\author{
Volker Konig, Renate Huch und Albert Huch
}

Klinik und Poliklinik für Geburtshilfe, Universitätsspital Zürich

Sollen Doppler-Ultraschall-Messungen umfassender und in einer anderen Weise ausgewertet werden, als cs dic in den Doppler-US-Geräten fest eingebauten Routinen erlauben, so müssen die interessicrenden Daten, das sind die sich aus der Fourieranalyse der Dopplershiftfrequenzen ergebenden Frequenzverteilungen, in einem externen Computer zugänglich sein. Im Folgenden wird ein Programmsystem beschrieben, das diese Datenübernahme während der DopplerUntersuchung durchführt und danach diese Daten auf verschiedene Arten auswerten kann: diverse Berechnungen der Maximal-Frequenzen (Hüllkurven), daraus resultierenden Berechnung der verschiedenen Indizes und insbesondere eine schnelle dreidimensionale Darstellung der Dopplerfrequenzen mit den Achsen Zeit, Frequenz und Intensität.

\section{Das Programmsystem DOP}

Voraussetzung für eine solche Auswertung ist, dass das verwendete Doppler-US-Gerät eine entsprechende Schnittstelle zur Datenübernahme aufweist. Dies ist allerdings nicht einmal bei allen Geräten der neuesten Generation der Fall.

Für das in unserer Klinik verwendete Gerät KRANZBUEHLER 8130 Duplex-Scanner wurde eine solche Schnittstelle in Zusammenarbeit mit dem Hersteller von uns eingebaut. Die Messungen mit dem ACUSON 128, der keine solche Schnittstelle aufweist, werden auf Video-Tape aufgenommen und dann nachträglich auf einen DOPTEK 8000 Frequenzanalyser, der serienmässig über eine entsprechende Schnittstelle verfügt, analysiert und in einen Computer überspielt. Als Computer dient ein IBM AT oder XT $286 \mathrm{mit}$ EGA-Grafik und einer I/O-Karte der Firma Metrabyte. Zum Einlesen, Darstellen, Auswerten und Speichern der Dopplerdaten wurde, zum grössten Teil in Pascal, ein Programmsystem geschrieben, bestehend aus einem Rahmenprogramm und diversen Unterprogrammen. Abb.1 zeigt das Hauptmenu, das die verschiedenen Anwendungsmöglichkeiten darstellt.

\section{Die Einleseprogramme}

Die erwähnten KRANZBUEHLER- und DOPTEK-Geräte führen 200 mal in einer Sekunde, d.h. alle $5 \mathrm{msec}$ eine Fourieranalyse durch, mit 80 Frequenzwerten zu je 64 Intensitätsstufen (6 Bit) in einem wählbaren Frequenzbereich von 2, 4, 8 oder $16 \mathrm{kHz}$, und zwar sowohl für Vorwärts-, wie für Rückwärtsgeschwindigkeiten. Auf dem Display des Gerätes erscheinen diese Frequenzwerte in einem Zeit/Frequenz Koordinatensystem, mit der Intensität als Farb- oder GrauwertKodierung. Werden die 6 Datenbits mit den zwei Steuerbits, die die Datenübergabe definieren, zu einem Byte zusammengefasst, so ergibt sich eine mittlere Uebertragungsrate von $38 \mathrm{kByte} / \mathrm{sec}$.

Programmpunkt 2 startet das Dateneinleseprogramm. Dieses liest die Daten für eine ausgewählte Richtung über die erwähnte $\mathrm{L} / \mathrm{O}-\mathrm{Karte}$ ein, entweder direkt während der Untersuchung oder aus dem Memory des DOPTEK-Gerätes. Dabei werden die Daten zyklisch in einen Array von $135 \mathrm{kByte}$, entsprechend einer Zeit von 9 Sekunden, eingeschrieben und gleichzeitig in Echtzeit zyklisch in 10 intensitätsgesteuerten Farbstufen auf dem Bildschirm gezeichnet, entsprechend der Darstellung auf dem DOPTEK. Da wegen der hohen Uebertragungsrate eine Verwendung von Interruptroutinen nicht möglich ist, werden die Daten durch Ansteuerung der EGARegister direkt in das EGA-Memory geschrieben. Dies alles erfordert für diesen Programmteil eine Programmierung in ASSEMBLER, mit einen zeitlich extrem optimierten Code, Speicherung der Startwerte der Bildschirmkoordinaten in Tabellen etc. Nach Abschluss der Aufnahme können die Daten sofort weiter ausgewertet werden und/oder es kann ein beliebig gewählter Teil der aufgenommenen 9 Sekunden in ein binäres File gespeichert werden.

Programmpunkt 1 liest die in einem solchen File gespeicherten Daten direkt in den Computer ein zur weiteren Darstellung und Auswertung. 
Die Auswerteprogramme

Es wurden für die verschiedenen Forschungs- und Anwendungszwecke Auswerteprogramme geschrieben. Das Hauptgewicht wurde dabei auf die Mittelung der vorliegenden Pulszyklen und auf eine dreidimensionale Darstellung des gesamten Frequenzmusters gelegt.

Für die Mittelung kann mit beweglichen Markern (auf Wunsch auch durch Maussteuerung) ein Bereich von maximal 9 Zyklen ausgewählt werden, in dem die Werte gemittelt werden sollen. Für diesen gemittelten Pulszyklus werden dann nach verschiedenen Algorithmen die Maximalfrequenzen und Medianwerte bestimmt. Hieraus können dann sowohl die gebräuchlichen Indizes berechnet werden, als auch für bestimmte Studien oder Untersuchungen die Berechnung spezieller Werte und Indizes einprogrammiert werden. (Zum Beispiel in Programmpunkt 6).

Eine schnelle "dreidimensionale Darstellung" der Dopplerfrequenzen mit den Achsen Zeit, Frequenz und Intensität (siehe Programmpunkt 5) ist ein wesentlicher Punkt des Auswerteprogramms. Der Aufbau eines solchen Spektrums, wie ihn Abb. 2 zeigt, dauert ca. 6 Sekunden. Diese dreidimensionalen Spektren werden bei uns benutzt für Studien zur Einbeziehung der Frequenzen kleiner als die Maximalfrequenzen in die diagnostischen Möglichkeiten, sowie zur Qualitätskontrolle der aufgenommenen Doppler-Werte.

Mit dem Programmteil 7 können, spezifisch für die jeweilige Anwendung, Indizes, Maximalfrequenzen usw. in ein File geschrieben werden, sodass eine bequeme Datenübernahme in andere Auswertesysteme wie dBase, Foxbase oder Statistikpakete gewährleistet ist.

\section{Zusammenfassung}

Zusammengefasst ergeben sich bei der Auswertung von Doppler-US-Messungen in einem externen Computer die folgenden Vorteile:

1) Dokumentation:

a) Messungen von maximal 9 Sekunden stehen reproduzierbar für weitere Auswertungen zur Verfügung.

b) Berechnete Werte können in Files geschrieben werden, spezifisch für eine einfache Datenübernahme in andere Systeme wie dBASE, EXCEL, Statistik-Pakete.

2) Auswertung der Maximal-Frequenzen:

a) Anwendung beliebiger Algorithmen zur Bestimmung der Maximal-Frequenzen.

b) Berechnung und Darstellung beliebiger Werte und Indizes, den jeweiligen Anwendungen oder Untersuchungen angepasst.

3) Dreidimensionale Darstellung:

a) Untersuchungen zur einfachen Beschreibung der Intensitätsverläufe.

b) Untersuchungen der hämodynamischen Bedeutung der kleineren Frequenzen und deren möglicher klinischer Relevanz.

c) Qualitätskontrolle der aufgenommenen Daten, auch für den Fall der reinen Maximal-Wert Bestimmung.

d) Untersuchung und Test der technischen Gerätedaten, wie Verstärkung,

Frequenzgang, Frequenzfilter etc.

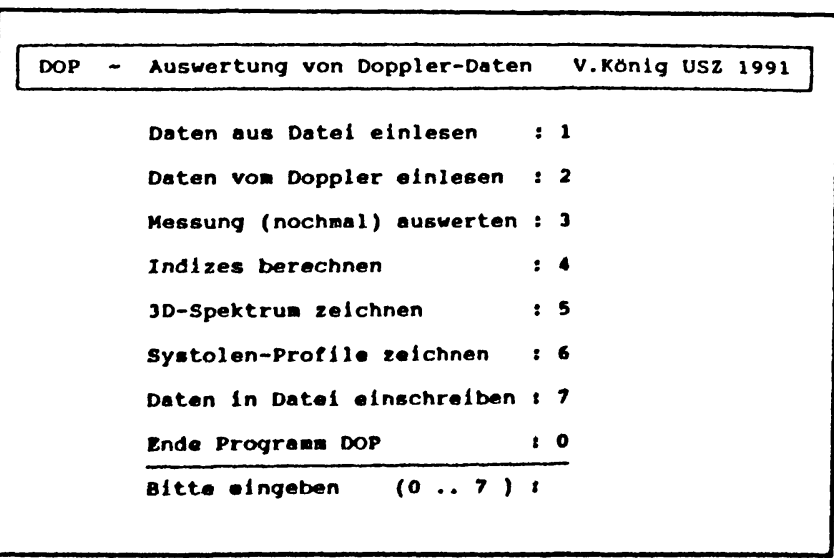

Abb.l: Hauplmenu des Programms fur die Auswertung von Doppler-Uluraschall-Messungen.

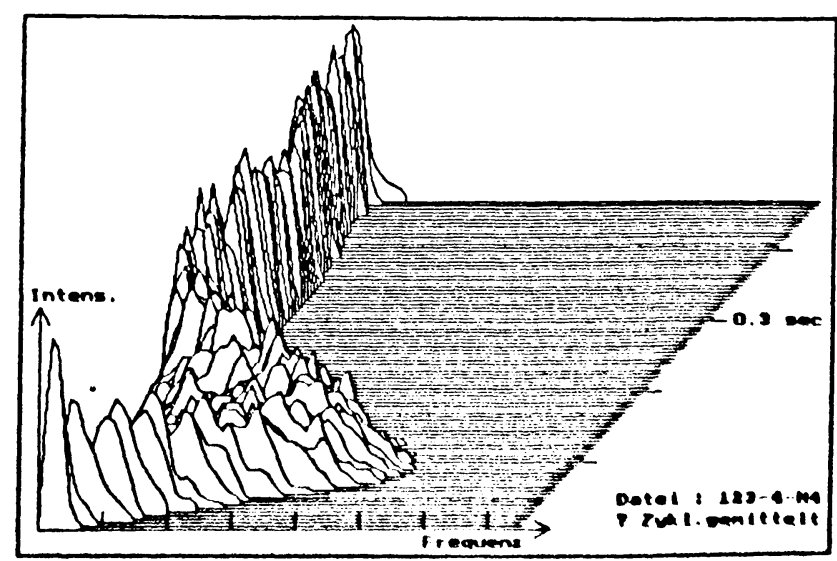

Abb.2: Das dreidimensionale Dopplerspehtrum einer NabelschnurAufnahme mit den Achsen Zeit, I'requenz und Intensitat. 